\title{
Adaptive Phenotypic Diversification along a Temperature-Depth Gradient
}

\author{
Jan Ohlberger, ${ }^{1,2, \star}$ Åke Brännström, ${ }^{2,3}$ and Ulf Dieckmann ${ }^{2}$ \\ 1. Department of Biology and Ecology of Fishes, Leibniz Institute of Freshwater Ecology and Inland Fisheries, D-12587 Berlin, Germany; \\ and Centre for Ecological and Evolutionary Synthesis, Department of Biosciences, University of Oslo, N-0316 Oslo, Norway; \\ 2. Evolution and Ecology Program, International Institute for Applied Systems Analysis, A-2361 Laxenburg, Austria; 3. Department of \\ Mathematics and Mathematical Statistics, Umeå University, SE-90187 Umeå, Sweden
}

Submitted November 2, 2012; Accepted March 18, 2013; Electronically published July 19, 2013

Online enhancements: appendixes.

\begin{abstract}
AвSTRACT: Theoretical models suggest that sympatric speciation along environmental gradients might be common in nature. Here we present the first data-based model of evolutionary diversification along a continuous environmental gradient. On the basis of genetic analyses, it has been suggested that a pair of coregonid fishes (Coregonus spp.) in a postglacial German lake originated by sympatric speciation. Within this lake, the two species segregate vertically and show metabolic adaptations to, as well as behavioral preferences for, correspondingly different temperatures. We test the plausibility of the hypothesis that this diversifying process has been driven by adaptations to different thermal microhabitats along the lake's temperature-depth gradient. Using an adaptive-dynamics model that is calibrated with empirical data and allows the gradual evolution of a quantitative trait describing optimal foraging temperature, we show that under the specific environmental conditions in the lake, evolutionary branching of a hypothetical ancestral population into two distinct phenotypes may have occurred. We also show that the resultant evolutionary diversification yields two stably coexisting populations with trait values and depth distributions that are in agreement with those currently observed in the lake. We conclude that divergent thermal adaptations along the temperature-depth gradient might have brought about the two species observed today.
\end{abstract}

Keywords: adaptive dynamics, ecological gradient, evolutionary diversification, sympatric speciation, temperature adaptation.

\section{Introduction}

Understanding the emergence of biological diversity by adaptive diversification based on natural selection is a major interest in evolutionary biology. The notion of adaptive speciation suggests that macroevolutionary phenomena of diversification are ultimately the outcome of microevolutionary processes driven by natural selection in general (Orr and Smith 1998; Coyne and Orr 2004) and by

\footnotetext{
* Corresponding author; e-mail: jan.ohlberger@bio.uio.no.

Am. Nat. 2013. Vol. 182, pp. 359-373. (c) 2013 by The University of Chicago. 0003-0147/2013/18203-54238\$15.00. All rights reserved.

DOI: $10.1086 / 671169$
}

frequency-dependent disruptive selection in particular (Dieckmann et al. 2004). From this notion, it follows that the formation of phenotypic and ecological diversity is likely to imply disruptive natural selection arising from competitive interactions. Competitive interactions between individuals and populations within the same geographical area-independently of whether they result from exploitation competition, interference competition, or apparent competition-are thus of major importance for understanding biological diversification. Empirical studies have confirmed that intraspecific competition can be frequency dependent (e.g., Schluter 2003; Swanson et al. 2003), making disruptive selection on corresponding traits of natural populations more likely than previously thought (Bolnick and Lau 2008). Frequency dependence occurs whenever selection pressures depend on the phenotypic composition of a population, which is a direct and often inevitable consequence of the way a population shapes the environment it in turn experiences.

The geographical conditions underlying diversifying processes have long been a focus of debate (e.g., Mayr 1963; Via 2001). However, the mechanisms of ecologically based sympatric, parapatric, and allopatric speciation that can drive divergence appear to be similar, with disruptive or divergent natural selection on ecologically important traits serving as the driving force of diversification and with the evolution of reproductive isolation occurring as a consequence of divergent selection on those traits (Schluter 2000). There is now mounting empirical evidence for the operation of ecological speciation in nature (Schluter 2009; Nosil 2012). The process of ecologically based adaptive speciation not only necessitates the emergence of reproductive isolation during diversification but also requires the ability of the incipient species to coexist persistently (Coyne and Orr 2004). Hence, the same ecological conditions and mechanisms that facilitate disruptive natural selection can cause adaptive speciation 
through gradually divergent evolution, promote reproductive isolation between the incipient species, and enable the coexistence of closely related species in sympatry. Highlighting one common mode of ecological speciation, empirical work suggests that competition-driven divergent resource or habitat use plays an important role in causing ecological diversification (Svanbäck and Bolnick 2007) and promoting sympatric speciation (e.g., Gíslason et al. 1999; Knudsen et al. 2006) within natural populations. With this study, we aim to identify ecological conditions that potentially drive adaptive diversifications in sympatry, as well as the underlying phenotypic traits that are subject to disruptive natural selection.

The theory of adaptive dynamics (Metz et al. 1992, 1996; Dieckmann and Law 1996; Geritz et al. 1998) has facilitated the construction of theoretical models investigating ecologically based processes of evolutionary diversification (Doebeli and Dieckmann 2005). Addressing this objective is aided by the assumption, often made in the theory of adaptive dynamics, that there is a sufficient separation of the timescales on which ecological change and evolutionary changes unfold. The resultant framework allows evaluation of the potential for evolutionary diversification in complex adaptive systems, with an emphasis on the ecological conditions promoting the corresponding selection pressures on specific adaptive traits of natural populations. Frequency-dependent selection on a slowly evolving quantitative trait of an asexually reproducing population leads to directional evolution along the local selection gradient until an evolutionarily singular strategy is reached. This singular strategy can be either a fitness maximum, and hence locally evolutionarily stable for a single morph, or a fitness minimum, and hence an "evolutionary branching point" that potentially leads to the splitting and subsequent divergence of two genetically distinct morphs. Adaptivedynamics models have repeatedly shown that many natural ecological settings are expected to imply evolution to such fitness minima, at which evolutionary branching may then occur that is based on frequency-dependent disruptive selection (as reviewed, e.g., in Dieckmann et al. 2004; see also Ito and Dieckmann 2007). In accordance with the majority of empirical examples of adaptive diversification, most of the existing adaptive-speciation models assume ecological specialization through resource partitioning as the key driver of diversification.

One conclusion from advanced adaptive-speciation models is that sympatric speciation is theoretically plausible and may thus be a common process in nature (e.g., Dieckmann and Doebeli 1999). However, theoretical speciation models are not easily evaluated in terms of biological plausibility and may lack ecological realism or accuracy in their assumptions about the properties of natural systems. For instance, the parameter regions in which evo- lutionary branching may occur in such models are usually only indirectly comparable among different models, and quantitative comparisons to natural systems are often difficult to make without referring to one particular natural system. Data-based models of adaptive diversification, utilizing empirically motivated and quantitative ecological assumptions, are therefore needed to evaluate the importance of these processes in nature.

The main purpose of this study is to develop such an empirically motivated and data-based model for adaptive diversification in a specific natural system that has already been well investigated (Coregonus spp. in Lake Stechlin, Germany). The model aims to describe the diversification of a single ancestral population into two stably coexisting populations by physiological adaptation to different thermal microhabitats along a temperature-depth gradient in a temperate freshwater lake. Our model does not consider the genetic architecture of the quantitative trait involved in this process and is not meant to examine the evolution of reproductive isolation. We rather examine whether the ecological conditions in our study lake allow for the evolution of an ancestral population toward a fitness minimum and subsequent evolutionary branching.

\section{Material and Methods}

\section{Model System}

Temperate freshwater fish occupying postglacial lakes are among the best model systems for studying adaptive diversification. Several taxa in these systems exhibit an ecological diversity consistent with processes of adaptive speciation (Schluter 2000), including the coregonids (reviewed in Hudson et al. 2007). In the deep, postglacial Lake Stechlin in Germany (maximum depth $69 \mathrm{~m}$ ), a pair of closely related coregonids coexists: common vendace (Coregonus albula L.) and endemic dwarf-sized Fontane cisco (Coregonus fontanae Schulz and Freyhof). The two species are easily distinguished by differential spawning times and show distinct morphological characteristics (Schulz and Freyhof 2003; Helland et al. 2009). Their sympatric evolution has been suggested on the basis of mitochondrial DNA and microsatellite analyses (Schulz et al. 2006). Recent amplified fragment length polymorphism analyses could neither confirm nor reject this hypothesis (Mehner et al. 2010b). The species differ in their average population depths within the pelagic zone, with $C$. fontanae being found deeper in the water column than $C$. albula throughout the year. This difference in depth distribution is associated with a difference in mean experienced water temperature (Helland et al. 2007; Mehner et al. 2010a).

Despite the depth segregation, the diet compositions of 
the species are rather similar, with a clear dominance of planktonic food (Helland et al. 2008). Hence, mechanisms reducing competition and thus potentially driving divergence between the species are not significantly related to diet. Instead, an important factor promoting ecological divergence between the species is a difference in temperature-dependent metabolic costs of swimming (Ohlberger et al. 2008b). This directly influences the competitive abilities of the two populations via their efficiency of foraging at a specific temperature, and thus depth. Furthermore, the temperature preferences of the two species correspond to the temperatures at which their net costs of swimming are minimized (Ohlberger et al. 2008c), which underscores the role of temperature as the predominant environmental factor shaping the divergence between the two coregonids. This setting offers a unique opportunity for investigating whether the observed conditions allow for ecological and evolutionary diversification of pelagic fish populations along the temperature-depth gradient of the lake.

\section{Model Description}

We modeled asexual fish populations competing for the same zooplankton resource with a depth-dependent carrying capacity along a temperature-depth gradient $T(x)$, where $T$ denotes temperature and $x$ denotes depth. The fish populations can adapt to different ambient temperature conditions, that is, to different locations along the depth axis, through adaptations in a one-dimensional quantitative trait, which specifies the temperature-dependent metabolic optimum, or optimum foraging temperature. For a fish morph $i$, with $i=1, \ldots, n$, this trait value is denoted by $T_{i}$. For describing the evolutionary dynamics of the trait values, we assume asexual reproduction, a low mutation probability, and a low standard deviation $\sigma$ of mutational steps, so that evolution follows the canonical equation of adaptive dynamics (Dieckmann and Law 1996) in conjunction with fitness-based conditions for evolutionary branching (Geritz et al. 1998), as specified in detail below. For a didactic introduction to, and further details on, the adaptive-dynamics approach, see, for example, Diekmann (2004).

The fish population dynamics are deterministic and structured with regard to depth, with $0<x<x_{\max }$. Exploitation competition for zooplankton, considered the predominant cause of competition among fish in our model, is logistic and occurs at each depth. The foraging efficiency $r\left(T, T_{i}\right)$ of a fish morph $i$, with $i=1, \ldots, n$, is assumed to decline with temperature $T$ on both sides around the morph-specific optimum foraging temperature $T_{i}$. The maximum zooplankton density is described by the depth-dependent carrying-capacity density $K(x)$, which declines monotonically with depth. The gain in biomass density $B_{i}(x)$ of morph $i$ at depth $x$, which includes fecundity, is proportional to the potential consumption rate and the equilibrium zooplankton density. Biomass loss, which includes mortality, arises from maintenance costs $m(T)$, which decrease monotonically with temperature. Therefore, the per capita growth rates $f_{i}(x)$, in terms of biomass, are given by the difference between per capita resource intake rates and per capita maintenance costs. We denote by $f_{i}$ the average growth of morph $i$ across all depths $x$. We further assume the rapid redistribution of individuals along the temperature-depth gradient following foraging dynamics, which in our model can be chosen from a continuum between random foraging and optimal foraging by varying a parameter $\alpha$. Individuals keep adjusting their depth according to their potential consumption rate, their potential predation risk, and resource availability.

In addition to the morph indices $i=1, \ldots, n$ for the $n$ resident morphs, we use $i=0$ for a rare mutant. The growth rate $f_{0}$ of such a rare mutant equals its invasion fitness (Metz et al. 1992), with its first derivative (often called selection gradient or fitness gradient) denoted by $g_{i}$ and its second derivative denoted by $h_{i}$. These derivatives of invasion fitness are taken with respect to the mutant trait value $T_{0}$ and are evaluated at the trait value of the resident with trait value $T_{i}$. Appendix A provides an overview of all variables, functions, and parameters used in this study.

\section{Model Dynamics}

The population dynamics are described by the change in total biomass of fish morph $i$ over time,

$$
\frac{\mathrm{d}}{\mathrm{d} t} B_{i}=f_{i} B_{i}
$$

where $B_{i}$ is the total biomass and $f_{i}$ is the average growth rate of morph $i$. The total biomass is given by the integral of biomass density from zero to maximum depth,

$$
B_{i}=\int_{0}^{x_{\max }} B_{i}(x) \mathrm{d} x,
$$

where $B_{i}(x)$ is the biomass density of morph $i$ at depth $x$. The average growth rate of morph $i$ is thus given by

$$
f_{i}=\frac{1}{B_{i}} \int_{0}^{x_{\max }} f_{i}(x) B_{i}(x) \mathrm{d} x,
$$

with

$$
f_{i}(x)=\lambda C\left(x, T_{i}\right) P_{\mathrm{eq}}(x)-m(T(x))
$$


where $\lambda$ is the conversion factor from zooplankton mass to fish mass (i.e., the energy-assimilation efficiency of the fish), $f_{i}(x)$ is the morph's per capita growth rate at depth $x, C\left(x, T_{i}\right)$ is the potential consumption rate of morph $i$ with trait $T_{i}$ at depth $x, P_{\text {eq }}(x)$ is the equilibrium zooplankton density at depth $x$, and $m(T)$ measures the temperature-dependent maintenance costs. We thus assume that feeding under natural conditions can be approximated by a linear relationship with resource density. We also assume fast resource dynamics, so that the zooplankton density is always near its equilibrium (app. B; apps. B and C are available online).

For the adaptive-foraging dynamics, we assume that an individual fish with trait $T_{i}$ adjusts its depth according to its potential consumption rate $C\left(x, T_{i}\right)$, the equilibrium zooplankton density $P_{\text {eq }}(x)$, and a foraging probability $F(x)$ that accounts for the depth-dependent risk of predation by piscivorous predators,

$$
B_{i}(x)=B_{i} \frac{\left(C\left(x, T_{i}\right) P_{\mathrm{eq}}(x) F(x)\right)^{\alpha}}{\int_{0}^{x_{\max }}\left(C\left(x^{\prime}, T_{i}\right) P_{\mathrm{eq}}\left(x^{\prime}\right) F\left(x^{\prime}\right)\right)^{\alpha} \mathrm{d} x^{\prime}} .
$$

Here, $\alpha$ is the degree of foraging optimality (with $\alpha=$ 0 representing random foraging and $\alpha \rightarrow \infty$ representing optimal foraging).

The expected evolutionary dynamics of the trait value $T_{i}$ describing the metabolic temperature optimum of morph $i$, is given by the canonical equation of adaptive dynamics,

$$
\frac{\mathrm{d}}{\mathrm{d} t} T_{i} \propto B_{i} g_{i}
$$

(Dieckmann and Law 1996), where $B_{i}$ is the equilibrium population size and $g_{i}$ is the first derivative of a mutant's invasion fitness, that is, the selection gradient. More details on the evolutionary analysis are provided under the corresponding heading below.

\section{Parameter Estimation}

Data sources for all parameter estimates are provided in appendix A. Figure 1 and appendix B provide details on the estimation of functions describing the temperaturedepth gradient, observed zooplankton density, potential consumption rate, foraging efficiency, capture success, maintenance costs, and foraging probability. These functions and parameters were estimated on the basis of observational data from Lake Stechlin, in conjunction with various laboratory measurements on the two coregonids. The foraging optimality $\alpha$ was estimated on the basis of distribution patterns of the two coregonids in Lake Stechlin. This parameter measures the degree to which individuals forage at their temperature optimum. In order to estimate the degree of foraging optimality in the natural system, we ran our model without evolutionary dynamics for the empirically determined temperature optima as fixed trait values and compared the resulting average depths with the measured year-round average depths of the Lake Stechlin coregonids (Helland et al. 2007). Figure B1 shows, as a function of $\alpha$, the sum of absolute values of the deviations of the two modeled population depths from the two observed average population depths in the natural system. A foraging optimality $\alpha$ of about 6 was found to offer the best approximation for the foraging behavior of these fish. We thus use this $\alpha$ value in our further analyses.

\section{Evolutionary Analysis}

We use a pairwise invasibility analysis to investigate the evolutionary dynamics in our system under the specific ecological conditions encountered in Lake Stechlin. Pairwise invasibility analysis assumes that any mutant introduced to the system first occurs at very low numbers and that the resident population has come sufficiently close to its demographic equilibrium at the time a mutant is introduced (van Tienderen and de Jong 1986; Metz et al. 1992).

To evaluate the potential for directional evolution on the adaptive trait of an established resident population, we need to calculate the invasion fitness of a mutant with a trait value that slightly differs from that of the resident (Metz et al. 1992). This invasion fitness is given by the sum of the mutant's growth rates across all depths, evaluated at the demographic equilibrium of the resident population (app. B). In case of positive invasion fitness, the mutant generically replaces the resident (Geritz et al. 2002) and the resident population's trait value shifts accordingly. This directional selection on the trait of a monomorphic resident population persists as long as the selection gradient

$$
g_{i}=\left.\frac{\partial}{\partial T_{0}} f_{0}\right|_{T_{0}=T_{i}}
$$

remains positive or negative. The point at which directional evolution comes to a halt is referred to as an "evolutionarily singular strategy" (Metz et al. 1996).

Once evolution has reached such a singular strategy, selection becomes either stabilizing or disruptive, depending on the local shape of the fitness landscape described by $f_{0}$ as a function of $T_{0}$. If the singular strategy is located at a local fitness maximum, no more invasion of any nearby mutant morph is possible, so that the singular strategy is evolutionarily stable. If the singular strategy is instead located at a local fitness minimum, it is evolutionarily 
unstable. This means that all nearby mutant morphs can invade the system and establish a second resident population, so that the system becomes dimorphic. A strategy at which selection becomes disruptive and to which directional evolution can nevertheless converge is known as an "evolutionary branching point" (Metz et al. 1992, 1996; Geritz et al. 1998). Evolutionary branching can occur if the second derivative of the invasion fitness,

$$
h_{i}=\left.\frac{\partial^{2}}{\partial T_{0}^{2}} f_{0}\right|_{T_{0}=T_{i}}
$$

is positive. An evolutionary branching point implies that a mutant can invade and stably coexist with the resident population. In other words, an evolutionary branching point can give rise to a protected dimorphism, in which each of the two morphs can invade the other.

The direction of dimorphic evolution after evolutionary branching is determined analogously to the monomorphic case. This is achieved by testing a system with two established residents for invasibility by a mutant. If a mutant close to one of the residents has positive invasion fitness, it successfully invades the system and replaces that resident. Such dimorphic directional evolution then proceeds until a strategy pair is reached at which the two selection gradients vanish. At this point, further evolutionary branching may occur, if at least one of the populations is situated at a fitness minimum. Otherwise, evolution comes to a halt.

\section{Sexual Reproduction}

We incorporate sexual reproduction into the model, following standard procedures (Roughgarden 1979; Bulmer 1980). In the asexual model, the phenotype is normally faithfully inherited from parent to offspring, except for mutations occurring with small probability. In contrast, in the sexual model, offspring phenotypes are always subject to variation resulting from genetic segregation and recombination. Also, mating is assortative, so that individuals pair up for reproduction on the basis of their similarity in trait value.

Specifically, an individual $j$ with trait value $T_{j}$ mates with another individual $k$ with trait value $T_{k}$ according to a Gaussian probability distribution around its trait value with standard deviation $\sigma_{\mathfrak{a}}$,

$$
K_{\mathrm{a}}\left(T_{j}, T_{k}\right)=\frac{\exp \left((-1 / 2)\left(T_{j}-T_{k}\right)^{2} / \sigma_{\mathrm{a}}^{2}\right)}{\sqrt{2 \pi} \sigma_{\mathrm{a}}} .
$$

Thus, $\sigma_{\mathrm{a}}$ measures the width of the mating kernel $K_{\mathrm{a}}$ and therefore determines the degree of assortment, with a higher $\sigma_{\mathrm{a}}$ corresponding to random mating and a lower $\sigma_{\mathrm{a}}$ corresponding to increasingly assortative mating. The probability that phenotype $j$ reproduces with phenotype $k$ further depends on the frequency $F_{k}$ of the latter in the population. Assuming a discretized trait space with a finite number $n$ of possible phenotypes, $T_{i}=1, \ldots, n$, the frequency $F_{k}$ is given by

$$
F_{k}=\frac{B_{k}}{\sum_{i=1}^{n} B_{i}} .
$$

To account for the effects of genetic segregation and recombination, the offspring trait value $T_{i}$ is drawn from the following probability density,

$$
K_{\mathrm{sr}}\left(T_{i}, T_{j}, T_{k}\right)=\frac{\exp \left((-1 / 2)\left(T_{j k}-T_{i}\right)^{2} / \sigma_{\mathrm{sr}}^{2}\right)}{\sqrt{2 \pi} \sigma_{\mathrm{sr}}},
$$

that is, from a Gaussian distribution around the midparental trait value $T_{j k}=\left(T_{j}+T_{k}\right) / 2$, with standard deviation $\sigma_{\mathrm{sr}}$. Thus, $\sigma_{\mathrm{sr}}$ measures the width of the segregationrecombination kernel (Roughgarden 1979), which describes the distribution of offspring traits for given parents. This approach assumes that the variation introduced by the segregation and recombination of genes is constant over time (Roughgarden 1979).

The probability density of being born with trait value $T_{i}$ for a given maternal trait value $T_{j}$ depends on the frequency distribution of phenotypes in the population, the degree of assortment, and the variation with which the offspring trait value is inherited from the parents,

$$
w\left(T_{i}, T_{j}\right)=Z_{i j}^{-1} \sum_{k=1}^{n} F_{k} K_{\mathrm{a}}\left(T_{j}, T_{k}\right) K_{\mathrm{sr}}\left(T_{i}, T_{j}, T_{k}\right),
$$

where the normalization constant $Z_{i j}=$ $\sum_{i, k=0}^{n} F_{k} K_{\mathrm{a}}\left(T_{j}, T_{k}\right) K_{\mathrm{sr}}\left(T_{i}, T_{j}, T_{k}\right)$ is chosen so that $\sum_{i=0}^{n} w\left(T_{i}, T_{j}\right)=1$. Hence, the probability of being born with trait value $T_{i}$ from all possible matings is given by

$$
w\left(T_{i}\right)=\sum_{j=1}^{n} F_{j} w\left(T_{i}, T_{j}\right)
$$

To determine whether the evolved trait distribution in the sexual model is unimodal (representing a single species) or bimodal (representing two specialist species), we directly compared the biomass densities of the phenotypic class adapted to $T_{i}=8.1^{\circ} \mathrm{C}$ (corresponding to the evolutionarily singular strategy in the asexual model) to those of the phenotypic classes adapted to $T_{i}=5^{\circ}$ and $10^{\circ} \mathrm{C}$ (corresponding to the two specialists at the evolutionary endpoint of the asexual model). When the intermediate class was less frequent than both the warm-adapted and the cold-adapted classes, we considered the outcome of the sexual model to represent two distinct morphs. As for the asexual model, we used numerical analyses to investigate the sexual model. 

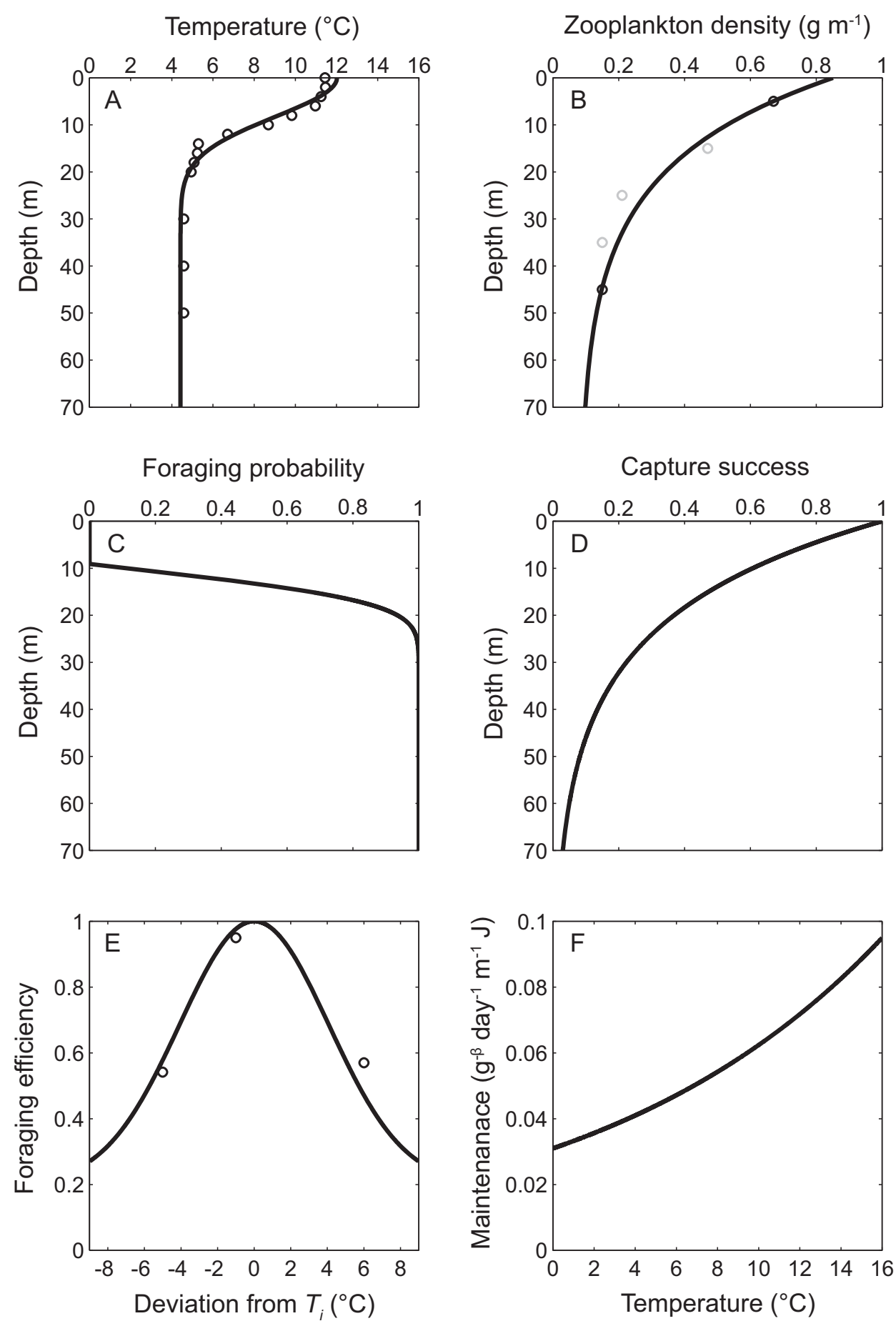

Figure 1: Functions used as model input (curves), together with the corresponding empirical data (circles). A, Temperature as a function of depth $\left(T(x)=T_{\min }+T_{\text {diff }} e^{-\phi x^{2}}\right)$. B, Zooplankton carrying-capacity density as a function of depth $\left(K(x)=Z_{\min }+Z_{\text {diff }} e^{-\varphi x}\right)$. C, Foraging probability as a function of depth $\left(F(x)=\left(T_{\max }-T(x)\right)_{+} /\left(T_{\max }-T_{\min }\right)\right)$. D, Capture success as a function of depth $\left(n(x)=e^{-\psi x}\right) ; E$, Foraging efficiency as a function of temperature $\left(r\left(T, T_{i}\right)=r_{\min }+\left(1-r_{\min }\right) e^{-v\left(T-T_{i}\right)^{2}}\right) . F$, Maintenance costs as a function of temperature $(m(T)=$ $\left.\gamma m_{0} B_{\text {mean }}^{\beta} e^{\omega T}\right)$. See appendixes $\mathrm{A}$ and $\mathrm{B}$, the latter available online, for further details. 


\section{Results}

To investigate the evolutionary dynamics of our asexual model and to answer the question of whether evolutionary branching may occur under the ecological conditions specified by the data-based parameter estimates and empirically motivated structural model assumptions, we apply the methods of evolutionary analysis described above. We visualize our results by using a pairwise invasibility plot (PIP) and a trait-evolution plot (TEP; Geritz et al. 1998). Furthermore, to investigate the dynamics of the sexual model, we evaluate how the evolutionary outcome is determined by the widths of the mating kernel (degree of assortment) and the segregation-recombination kernel (offspring distribution).

Figure 2 shows the PIP for all trait-value combinations of a resident and a mutant morph with temperature optima between $4^{\circ}$ and $12^{\circ} \mathrm{C}$ for a foraging optimality $\alpha$ of 6. A monomorphic population starting with any trait value is subject to directional selection and therefore evolves until the singular strategy is reached at about $8.1^{\circ} \mathrm{C}$. At this point, the population experiences a fitness minimum and selection becomes disruptive, so that evolutionary branching can occur.

Figure 3 provides a TEP to visualize the subset of trait combinations for which the two morphs are able to coexist in a protected dimorphism (gray area). It also depicts the direction of selection gradients (arrows), the evolutionary isoclines along which one of the selection gradients vanishes (solid and dotted lines), and the expected course of dimorphic directional evolution after evolutionary branching (dashed lines). Within the area of coexistence, the two morphs evolve to a point (large filled circles) at which the two isoclines intersect. The resultant trait combinations are $5.0^{\circ}$ and $10.0^{\circ} \mathrm{C}$. At these trait combinations, both selection gradients vanish and directional selection for the two morphs thus ceases. Since both isoclines are at a local fitness maximum at their intersection, the resulting dimorphism is locally evolutionarily stable. Hence, no secondary evolutionary branching can take place in the system, and the two morphs are recognized as the modelpredicted evolutionary outcome.

Figure 4 compares the model-predicted trait values and biomass distributions with the empirically observed trait values and biomass distributions. Figure $4 A$ shows time series of the evolving monomorphic and dimorphic optimum foraging temperatures and compares the latter with the observed temperature preferences of the two coregonids in Lake Stechlin. The initial trait value of the monomorphic population does not alter the evolutionary outcome, since the evolutionary branching point is globally convergence stable (i.e., a monomorphic population evolves toward this point irrespective of its initial value).

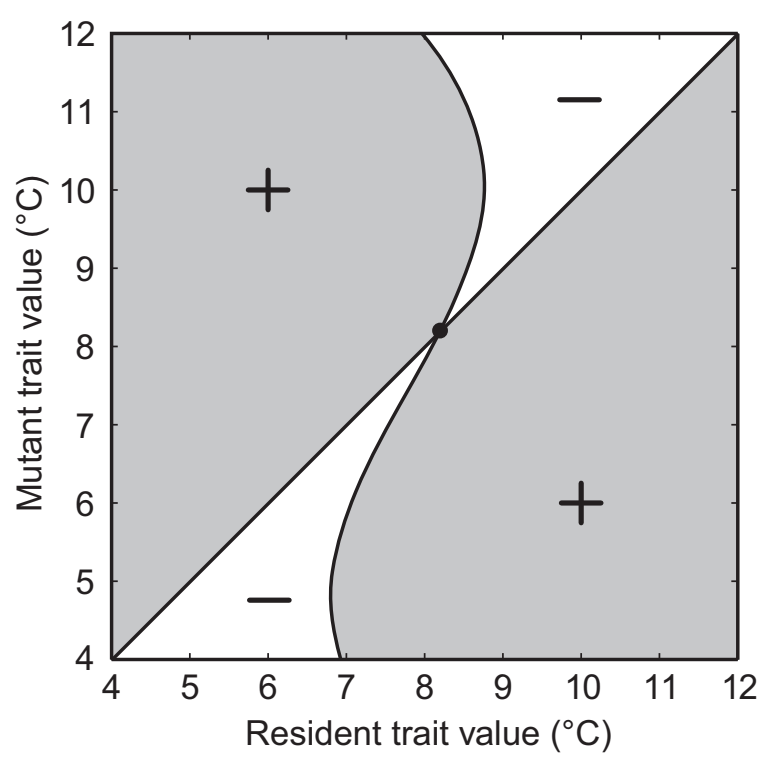

Figure 2: Pairwise invasibility plot (PIP) for trait values between $4^{\circ}$ and $12^{\circ} \mathrm{C}$. The gray areas indicate trait-value combinations at which a mutant phenotype can invade a resident phenotype, that is, where the mutant's invasion fitness is positive. The evolutionarily singular strategy at which the selection gradient vanishes is indicated by the small filled circle. On the basis of this PIP, we expect that a monomorphic population continuously evolves along the main diagonal until it reaches the evolutionarily singular strategy, at about $8.1^{\circ} \mathrm{C}$ (which implies that the singular strategy is convergence stable). At this branching point, the population experiences a fitness minimum, and selection accordingly becomes disruptive (which implies that the singular strategy is locally evolutionarily unstable).

The temperature optima at the endpoint of dimorphic evolution $\left(5.0^{\circ}\right.$ and $\left.10.0^{\circ} \mathrm{C}\right)$ are in good agreement with the measured temperature preferences of the two coregonids $\left(4.2^{\circ}\right.$ and $9.0^{\circ} \mathrm{C}$; Ohlberger et al. $\left.2008 \mathrm{c}\right)$. Figure $4 B$ shows the depth distributions of the two morphs after dimorphic evolution has come to a halt and compares their average depths with the year-round average depths observed for the coregonids in Lake Stechlin. This comparison shows that the model-predicted average depths of the populations at the endpoint of dimorphic evolution match very well those observed in the field (Helland et al. 2007).

Figure 5 displays the evolutionary outcome for the sexual model in dependence on the functions describing assortative mating and segregation/recombination. The diagram shows that speciation becomes more likely as the standard deviations of these two kernels decrease. For low degrees of assortment (high $\sigma_{\mathrm{a}}$ ) and for a wide offspring trait distribution around the midparental trait value (high $\left.\sigma_{\text {sr }}\right)$, the population remains monomorphic, centered around a trait value of about $8^{\circ} \mathrm{C}$. The threshold for the width of the assortative-mating kernel above which di- 


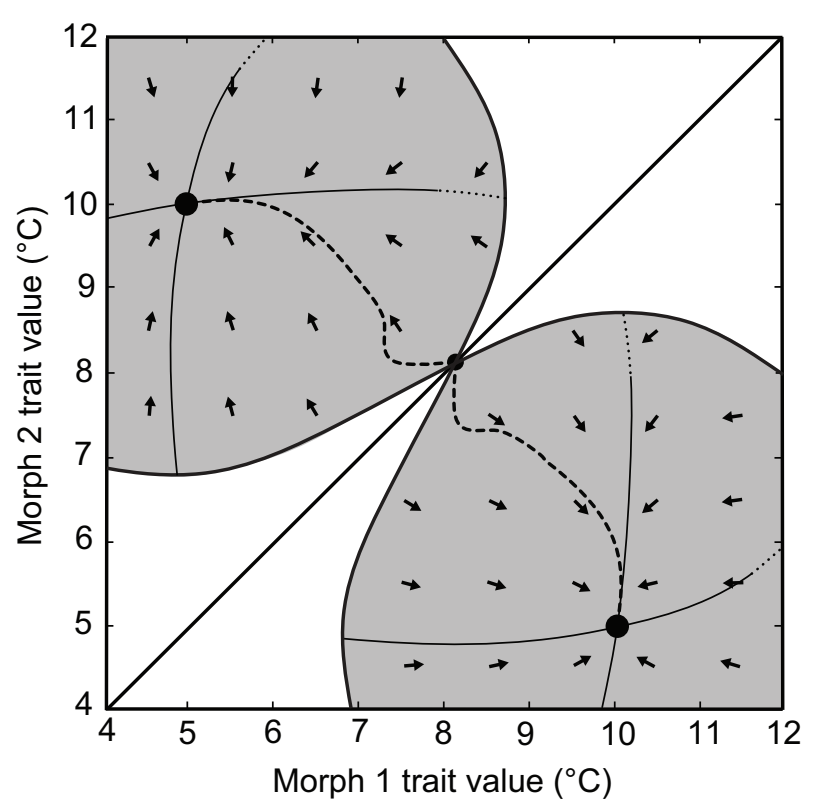

Figure 3: Trait-evolution plot (TEP) for trait values between $4^{\circ}$ and $12^{\circ} \mathrm{C}$. The parts of this plot that lie above and below the diagonal are fully equivalent, since the numbering of the two considered morphs is arbitrary. The gray areas represent trait-value combinations for which the two morphs can stably coexist, that is, for which they form a protected dimorphism. Arrows indicate the direction of the selection gradient within this coexistence area. The solid and dotted lines show the evolutionary isoclines, along which the selection gradient for one morph vanishes. As explained in figure 2, evolutionary branching can occur at the small filled circle. The dashed lines show the evolutionary dynamics after branching, which converge to the protected dimorphism at which the two evolutionary isoclines intersect (large filled circles). Since this intersection point is located along the parts of the isoclines that correspond to a fitness maximum (solid lines), as opposed to a fitness minimum (dotted lines), the resulting dimorphism is not only protected but also locally evolutionarily stable. The two thus established resident morphs have trait values of $5.0^{\circ}$ and $10.0^{\circ} \mathrm{C}$.

versification cannot occur is $\sim 0.9$, which means that a focal individual choosing between two potential mates is $65 \%$ as likely to choose a mate whose trait value differs by $1^{\circ} \mathrm{C}$ as to choose a mate with its own trait value. The threshold for the width of the segregation-recombination kernel above which diversification cannot occur is $\sim 0.6$, which means that the offspring trait value has a probability of $\sim 90 \%$ of differing by less than $1^{\circ} \mathrm{C}$ from the midparental trait value. Our results show that the two thresholds for the assortative-mating kernel and the segregation-recombination kernel are fairly independent of each other.

Figure 6 shows a trait-evolution plot for the sexual model for a given degree of assortment $\left(\sigma_{\mathrm{a}}=0.2\right)$ and a given variance of the offspring trait distribution $\left(\sigma_{\mathrm{sr}}=\right.$ 0.2 ). This setting yields a dimorphic outcome, with the highest biomass densities at trait values around $5^{\circ}$ and $10^{\circ} \mathrm{C}$, which is in accordance with the values we have identified above for the asexual model and the values that have been reported for the empirically observed thermalpreference traits of the two species (Ohlberger et al. 2008c).

A main target parameter for a sensitivity analysis of our model is the foraging optimality $\alpha$, since the evolutionary dynamics are much affected by this parameter. An $\alpha$ value of less than about 1 results in a monomorphic evolutionarily stable strategy at an intermediate temperature optimum, whereas values higher than about 10 may lead to secondary evolutionary branching and thus to the evolutionary establishment of higher degrees of polymorphism. It is therefore reassuring to confirm that even considerable variations in $\alpha$ (over the range 1-10, compared with the value of $\alpha=6$ estimated from the empirical distribution patterns of the two coregonids in the lake; fig. B1) do not have a qualitative effect on the evolutionary outcome in our model. We note, however, that as higher values of $\alpha$ approximate the foraging behavior of the fish reasonably well (fig. $1 B$ ), the ecological conditions in the lake might favor multiple evolutionary branching. In that case, other factors, such as the degree of assortment or the distribution of offspring trait values, could have prevented a second diversification event among the studied coregonids. All other model parameters are based on empirical data from Lake Stechlin and the species pair, are direct system characteristics, or have been taken from other literature on coregonids (app. A). In order to account for uncertainty in the empirically derived parameters and to check the robustness of our results to changes in these parameters, we performed a univariate sensitivity analysis by increasing or decreasing each parameter value by $10 \%$ and evaluating the resulting trait values at the evolutionary endpoint of the asexual model (table C1, available online). All considered parameter perturbations allow for evolutionary branching and result in trait values for the two morphs that are similar to those predicted by the nonperturbed model — which means that they are also similar to the experimentally determined trait values of the two coregonids. The trait value of the cold-adapted phenotype $\left(4.6^{\circ}-5.5^{\circ} \mathrm{C}\right)$ was most strongly affected by changes in the minimum temperature at lake bottom $\left(T_{\min }\right)$, that is, by the lower thermal limit of the habitat available to the fish. The trait value of the warm-adapted phenotype $\left(9.1^{\circ}-10.8^{\circ} \mathrm{C}\right)$ was most strongly affected by changes in the maximum temperature at which the fish forage $\left(T_{\max }\right)$, that is, by the upper thermal limit of their habitat (app. C).

\section{Discussion}

It is widely believed that the same ecological conditions that produce disruptive selection and cause adaptive spe- 

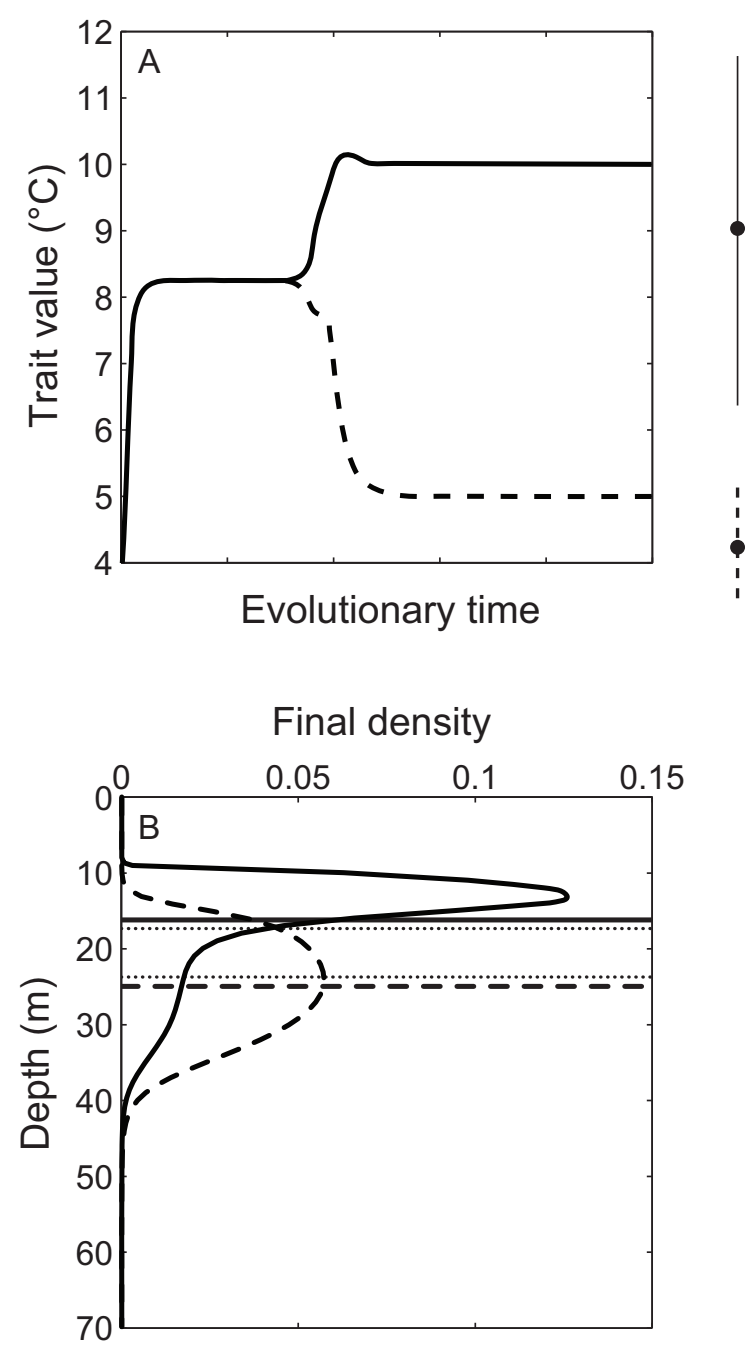

Figure 4: Comparison of model predictions and observations of the natural system. A, Evolution of trait values over time (solid and dashed lines), in comparison with the trait values observed for the natural populations. The monomorphic evolution (left part) is represented by the pairwise invasibility plot in figure 2 , whereas the dimorphic evolution after branching (right part) is represented by the trait-evolution plot in figure 3. The initial optimum foraging temperature for the monomorphic population (here set to $4^{\circ} \mathrm{C}$ ) can be randomly chosen, since it does not affect the evolutionary outcome of the model. The evolutionary outcome for the optimum foraging temperatures of the two resulting morphs $\left(5.0^{\circ}\right.$ and $\left.10.0^{\circ} \mathrm{C}\right)$ is in good agreement with the experimentally determined temperature preferences of the Lake Stechlin coregonids. These are shown as point estimates with $95 \%$ confidence intervals on the right side of the plot for Coregonus fontanae (dashed line) and Coregonus albula (continuous line; Ohlberger et al. 2008c). Units along the horizontal axis can be considered arbitrary, as they are freely adjustable through the choice of mutational parameters. $B$, Depth distributions at the evolutionary endpoint (solid and dashed curved lines) for the two populations. The resultant model-predicted average depths (solid and dashed horizontal lines) are compared with the average depths observed for the Lake Stechlin coregonids (dotted lines), showing very good agreement (at 17 and 24 m, respectively; Helland et al. 2007). ciation drive the ecological differentiation that enables the coexistence of closely related species in sympatry (Schluter 2000; Coyne and Orr 2004). Previous empirical studies on the species pair in Lake Stechlin had revealed that the two coexisting coregonids have diverged with respect to their vertical distribution in the lake (Helland et al. 2007, 2009), the temperature dependence of their metabolisms (Ohlberger et al. 2008b), and their associated thermal preferences (Ohlberger et al. 2008c). The concordance in the ecological, physiological, and behavioral differentiation of the species with respect to temperature suggests thermal specialization as the main driver of their eco-evolutionary divergence. This divergence may have originated from two preexisting species through character displacement or from a single ancestral species through sympatric speciation, with the latter option being supported by genetic analyses (Schulz et al. 2006; Mehner et al. 2010b).

We have shown, by analyses of the evolutionary dynamics of the model introduced in this study, (1) that under the specific empirical conditions observed in Lake Stechlin, evolutionary branching of a single ancestral population into two morphs with distinct thermal specialization should have been favored by the ecological conditions, (2) that the two

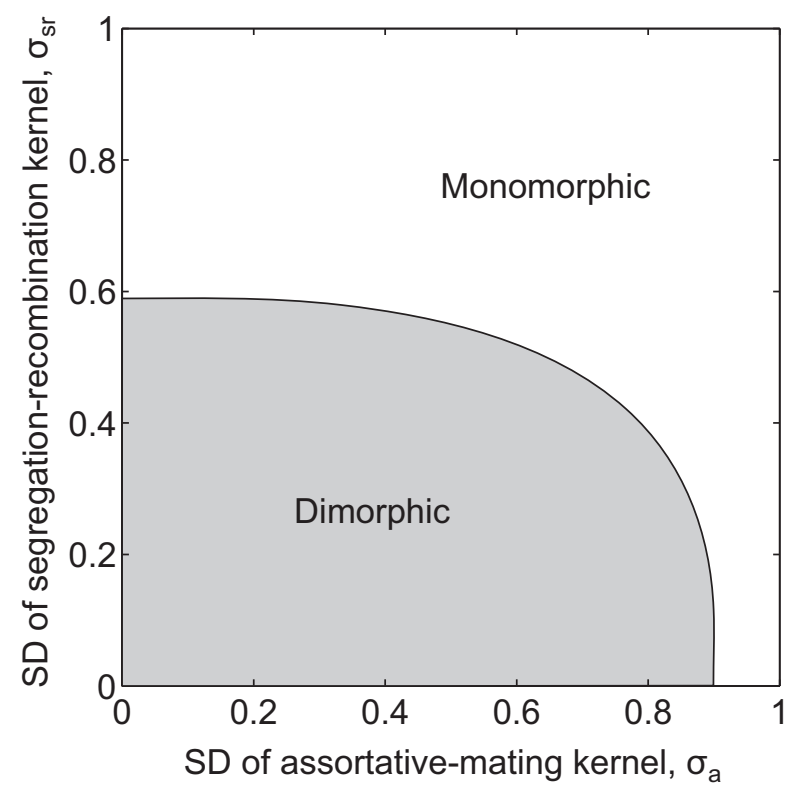

Figure 5: Evolutionary outcomes of the sexual model (white for monomorphic, gray for dimorphic), depending on the widths of the assortative-mating kernel $\left(\sigma_{\mathrm{a}}\right)$ and of the segregation-recombination kernel $\left(\sigma_{\mathrm{sr}}\right)$. The number of morphs represented by the resulting trait distribution for a given combination of $\sigma_{\mathrm{a}}$ and $\sigma_{\mathrm{sr}}$ is determined by comparing the biomass density of the single phenotype representing the evolutionarily singular strategy (trait value $8.1^{\circ} \mathrm{C}$ ) to the sum of the biomass densities of the two phenotypes representing the evolutionary endpoints of the asexual model (trait values $5^{\circ}$ and $10^{\circ} \mathrm{C}$ ). 


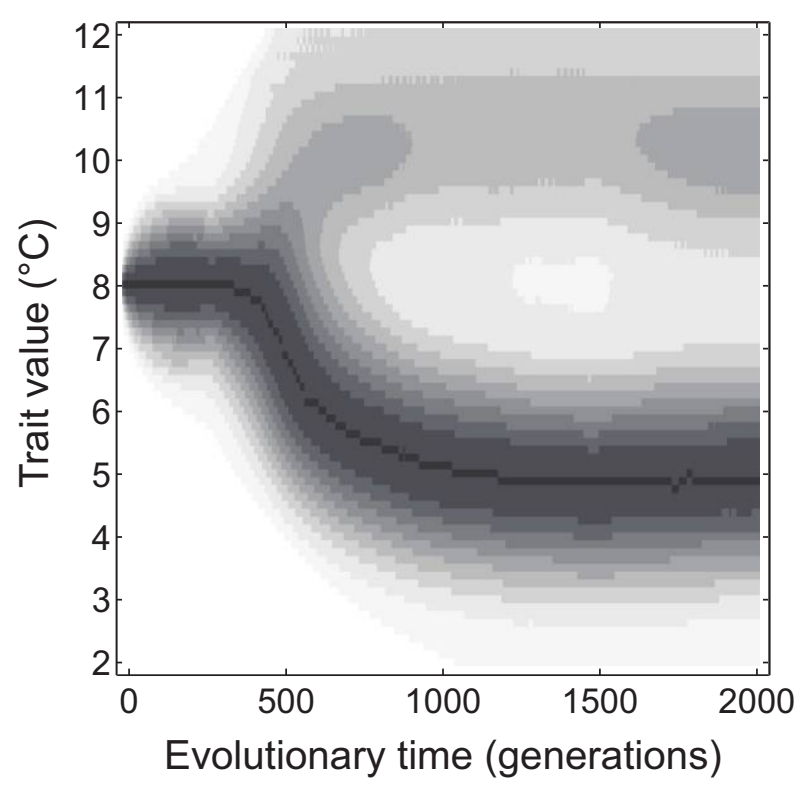

Figure 6: Trait-evolution plot for the sexual model, illustrating the distribution of biomass density across all phenotypes (dark gray for high biomass density, light gray for low biomass density). The parameters for the two kernels are $\sigma_{\mathrm{a}}=0.2$ and $\sigma_{\mathrm{sr}}=0.2$, and the resolution in trait space was set to a value of 0.1 . The sexual model yields a dimorphic outcome similar to that of the asexual model, with the highest biomass densities at trait values around $5^{\circ}$ and $10^{\circ} \mathrm{C}$.

model-predicted populations can stably coexist, and (3) that they have temperature optima and depth distributions that closely correspond to those in the field. The model thereby supports the hypothesis that the temperature-depth gradient in this system has mediated the thermal specialization of the two species, thereby allowing them to occupy different thermal niches along the vertical lake axis. This result clearly contrasts with the commonly observed divergence of many other sympatric fish pairs, especially in newly colonized postglacial lakes. The common situation in these lakes is the occurrence of limnetic-benthic species pairs that have diverged into distinct ecotypes or species by exploiting either benthic food in profundal or littoral habitats or planktonic food in pelagic habitats, giving rise to an ecological pattern known as trophic polymorphism (Schluter and McPhail 1993; Lu and Bernatchez 1999; Knudsen et al. 2006). Our results are in line with recent findings suggesting that divergent thermal adaptation of sibling taxa caused by differential selection between thermal environments may underlie reproductive isolation or allow for stable coexistence in several animal and plant systems (Keller and Seehausen 2012).

To our knowledge, we present the first data-based model of adaptive diversification along a continuous environmental gradient. On the basis of our model analyses, we conclude that the ecological conditions in Lake Stechlin are prone to evolutionary branching and that sympatric speciation of coregonids in this lake is thus an ecologically plausible scenario. Since the hypothesized speciation process occurs along an environmental gradient, that is, along a spatial dimension in our model, it possesses elements of parapatric speciation. However, since isolation by distance is not expected to play any significant role over the short spatial ranges that characterize the vertical differentiation of the two species, the particular speciation process modeled here presumably is very close to the sympatric end along the sympatric-allopatric continuum of parapatric speciation. Other authors who have attempted to describe the evolutionary dynamics of a particular system on the basis of empirical data have used models in which selection (acting on several traits) is density dependent and the environment is represented by a number of novel and discrete ecological niches (e.g., Gavrilets and Vose 2007; Gavrilets et al. 2007). The main advantage of an empirically calibrated mathematical model of adaptive speciation is its ability to help identify the crucial environmental factors in processes of evolutionary diversification. Previously, the theory of adaptive dynamics has been successfully applied to developing various strategic models of sympatric and parapatric speciation, although its usefulness for approaching evolutionary problems has occasionally been challenged (e.g., Gavrilets 2005; Waxman and Gavrilets 2005). Our work here, although limited by the underlying simplifying assumptions and by a focus on one specific natural system, shows that adaptive-dynamics theory also offers useful tools for identifying, through interfacing with the relevant empirical data, key ecological factors involved in processes of evolutionary diversification under natural conditions.

The endpoint of evolutionary dynamics in our model describes a dimorphic fish population consisting of one morph that has a lower temperature optimum and occupies deeper water layers relative to another morph that has a higher temperature optimum and occupies shallower water layers (with optimum foraging temperatures of $5.0^{\circ}$ and $10.0^{\circ} \mathrm{C}$, respectively). This model outcome matches the experimentally determined species-specific thermal preferences and corresponding temperatures of lowest swimming costs $\left(4.2^{\circ}\right.$ and $9.0^{\circ} \mathrm{C}$; Ohlberger et al. $2008 b$, $2008 c$ ) and is in good accordance with the experienced water temperatures of the coregonids in Lake Stechlin $\left(4.0^{\circ}-6.0^{\circ}\right.$ and $6.5^{\circ}-9.0^{\circ} \mathrm{C}$; Mehner et al. 2010a). These comparisons show that the two populations that evolve in our model represent well the natural populations in terms of their distributions and trait values. Interestingly, Lake Stechlin and the nearby Lake Breiter Luzin are the only two lakes in Germany in which sympatric pairs of coregonids occur. Both are located at the southern border of 
the Coregonus albula distribution range (Kottelat and Freyhof 2007) and are the deepest in that region (Mehner et al. 2005a), thus featuring the broadest temperature ranges and steepest gradients among lakes worldwide in which C. albula occurs (Kottelat and Freyhof 2007). Besides temperature, light intensity decreases continuously with lake depth. This might have facilitated a differentiation in lightdependent feeding efficiencies along the depth gradient. However, there is good evidence that the two species do not differ in their foraging efficiency at light intensities experienced in the lake (Ohlberger et al. 2008a). Other factors that might have facilitated the evolutionary divergence of an ancestral population include a species-poor environment and a high level of intraspecific competition at the time of colonization, conditions that are often found in newly colonized postglacial lakes and are believed to promote ecologically based adaptive diversification in fishes (Vamosi 2003; Bolnick 2004).

Environmental temperature gradients are common phenomena in nature, with a major effect on biogeographical patterns of species distributions. For instance, the largescale biogeography and the small-scale distribution of fishes are broadly shaped by temperature patterns (Somero 2002, 2005; Pörtner et al. 2007, 2010). Furthermore, it has been reported that niche segregation of fishes can be facilitated by differentiation in thermal preference (Larsson 2005) and that adaptive differences in thermal physiology promote ecological divergence between closely related intertidal fishes (Hilton et al. 2008). However, the general importance of environmental gradients for small-scale patterns of species distributions and their diversification has long been a matter of debate. The traditional understanding is that gradual evolution of a quantitative trait of a single population along an environmental gradient would necessarily result in local adaptation to an optimum, with some smooth and continuous variation around this optimum due to the homogenizing effects of gene flow and stabilizing selection (e.g., Kirkpatrick and Barton 1997). More recent theoretical models, however, show that frequency-dependent selection due to local competition in trait space and physical space naturally results in discrete clustering of an adaptive trait along a continuous environmental gradient: these models demonstrate the plausibility of evolutionary branching under such ecological conditions in asexual populations (Mizera and Meszéna 2003; Leimar et al. 2008), as well as the evolution of reproductive isolation in sexual populations (e.g., Doebeli and Dieckmann 2003; Heinz et al. 2009). Interestingly, empirical evidence for the maintenance and emergence of adaptive divergence along environmental gradients is accumulating, in particular relating to depth gradients of aquatic environments (Vonlanthen et al. 2009; Ingram 2011). The results presented here support the theory of ecologically based adaptive diversifications along environmental gradients. The two populations segregate in space, even though the underlying spatial range is continuous and does not feature any intrinsic bimodality of environmental conditions.

There is ongoing debate among evolutionary ecologists as to whether adaptive, sympatric speciation is a plausible and common scenario for the evolution of biological diversity (e.g., Bolnick and Fitzpatrick 2007; Räsänen and Hendry 2008). Such a process requires the evolution of two ecologically distinct and stably coexisting populations from a common ancestor as well as the emergence of reproductive isolation between the diverging populations. The purpose of our model was to identify potential ecological drivers and physiological determinants of the diversifying process in a well-studied natural system. Our results suggest that adaptive diversification would likely be promoted by natural selection under the specific ecological conditions studied through our model. We found that evolutionary diversification in the sexual model is likely if the probability of choosing the same phenotype over a phenotype that differs in trait value by $1^{\circ} \mathrm{C}$ is greater than $65 \%$ (corresponding to $\sigma_{\mathrm{a}}=0.9$ ). To our knowledge, no empirical estimates exist of mating probabilities based on traits related to thermal preference/performance of fishes within a similar ecological context. Yet some degree of phenotype matching seems likely for the coregonids, considering that changes in temperature preference are associated with changes in habitat use and, potentially, in growth efficiency (Mehner et al. 2011). We also found that diversification in the sexual model is likely if the probability that the offspring trait value differs by less than $1^{\circ} \mathrm{C}$ from the midparental trait value is greater than $90 \%$ (corresponding to $\sigma_{\mathrm{sr}}=0.6$ ). The resulting standing variation in a hypothetical monomorphic population corresponds to a genetic coefficient of variation of $13 \%$ (Houle 1992). For comparison, a value of $6 \%$ was used for the initial genetic coefficient of variation in an evolutionary model parameterized for Atlantic cod (Gadus morhua), a value that was considered conservative (i.e., low) in light of the available empirical evidence (Enberg et al. 2009).

Reproductive isolation between the Lake Stechlin coregonids is attained via a phenological separation in terms of spawning times, with $C$. albula spawning in late autumn and Coregonus fontanae in spring (Schulz and Freyhof 2003). This temporal separation of reproduction, which hinders gene flow between the populations, might have evolved as a by-product of thermal specialization, owing to slower maturation and later spawning at lower temperatures. In fact, the majority of other European populations of the C. albula complex are autumn spawners (Kottelat and Freyhof 2007; Mehner et al. 2010b) and generally show thermal preferences far above that of $C$. fontanae in Lake Stechlin (Ohl- 
berger et al. 2008c). Another potential by-product of such a thermal adaptation is differentiation in body size, as observed for the coregonids in Lake Stechlin. Mature females of $C$. fontanae are smaller (87-126 mm standard length) than those of sympatric C. albula (134-167 mm standard length), with both maturing at about one-and-a-half years (Schulz and Freyhof 2003). Colder water temperatures slow the rate of energy assimilation and hence that of growth and maturation (Mehner et al. 2011). In consequence, coldadapted phenotypes are more likely to mature later than warm-adapted ones, which may lead to a phenological separation in spawning time. This assortment may be reinforced by the lack of food during winter months, which can impede maturation and thus lead to a further delay in maturation until spring. Such a bimodal spawning opportunity would strengthen assortment based on similarity in optimum temperature. Reproductive isolation through assortative mating based on differences in spawning time and/ or location, possibly in combination with differentiation in body size, has been described for many species, including freshwater fishes in postglacial lakes (Jonsson and Jonsson 2001; Østbye et al. 2004). It should also be noted, however, that whether assortative mating preceded or followed ecological divergence remains uncertain in most cases (Snowberg and Bolnick 2008).

While other mechanisms, in principle, might have brought about the temporal separation in spawning times, there are no data supporting this view. Furthermore, the evolution of reproductive isolation is not sufficient to explain the stable coexistence of species if these are ecologically identical (Coyne and Orr 2004). Because the two Lake Stechlin coregonids have surprisingly similar diets, their differential use of thermal microhabitats appears to be the only mechanism by which they have achieved ecological segregation. Whether divergence in thermalmicrohabitat use and the corresponding adaptations caused a shift in spawning time or whether causation has worked in the opposite direction cannot be answered with present knowledge. It should also be acknowledged that the ecological conditions in the lake have not been stable since the last glaciation and that past climate events might have facilitated the segregation.
Our study highlights the potential role of temperature gradients for shaping processes of ecological and evolutionary diversification through thermal adaptation. This perspective on the ecologically based evolution of sympatric species is currently receiving increasing scientific interest and presents a promising path for future research (Keller and Seehausen 2012; Svensson 2012). Prospective work may use our model as a starting point, for example, to develop a predictive evolutionary framework for evaluating the impact of future temperature changes due to global warming on fish populations similar to those studied here. Such an approach could provide insights into the potential implications of thermal specialization for species interactions and for their adaptive responses to altered environmental conditions.

\section{Acknowledgments}

F. Hölker and T. Mehner provided valuable suggestions on the study design and on an earlier version of this manuscript. We thank I. P. Helland for providing unpublished data. We would also like to thank L. M'Gonigle and J. L. Payne for valuable discussions. Anonymous reviewers provided constructive comments that helped to improve the manuscript. Analyses of empirical data from Lake Stechlin were financed by a grant from the AQUASHIFT priority program of the German Research Council (DFG; grants Me 1686/5-1 and 5-2). J.O. received a grant from the German National Member Organization of the International Institute for Applied Systems Analysis for participating in the Young Scientists Summer Program (YSSP). U.D. gratefully acknowledges financial support from the European Commission, through the Marie Curie Research Training Network on Fisheries-Induced Adaptive Changes in Exploited Stocks (FishACE; grant MRTN-CT-2004005578) and the Specific Targeted Research Project on Fisheries-Induced Evolution (FinE; grant SSP-2006044276), under the European Community's Sixth Framework Program. U.D. received additional support from the European Science Foundation, the Austrian Science Fund, the Austrian Ministry of Science and Research, and the Vienna Science and Technology Fund. 


\section{APPENDIX A}

\section{Model Variables, Functions, and Parameters}

Table A1: Model variables and functions

\begin{tabular}{|c|c|c|}
\hline Notation & Unit & Description \\
\hline$x$ & $\mathrm{~m}$ & Depth \\
\hline$T(x)^{\mathrm{a}}$ & ${ }^{\circ} \mathrm{C}$ & Temperature at depth $x$ \\
\hline$T_{i}$ & ${ }^{\circ} \mathrm{C}$ & Temperature optimum of morph $i$ (evolving trait value) \\
\hline$i$ & Not applicable & Morph index $(i=1, \ldots, n$ : residents; $i=0$ : rare mutant $)$ \\
\hline$P(x)$ & $\mathrm{g} \mathrm{m}^{-1}$ & Zooplankton density at depth $x$ \\
\hline$P_{\mathrm{eq}}(x)$ & $\mathrm{g} \mathrm{m}^{-1}$ & Equilibrium zooplankton density at depth $x$ \\
\hline$K(x)^{\mathrm{a}}$ & $\mathrm{g} \mathrm{m}^{-1}$ & Carrying capacity of zooplankton at depth $x$ \\
\hline$C\left(x, T_{i}\right)^{\mathrm{a}}$ & $\mathrm{s}^{-1}$ & Potential consumption rate of morph $i$ with trait $T_{i}$ at depth $x$ \\
\hline$r\left(T, T_{i}\right)^{\mathrm{a}}$ & Not applicable & Foraging efficiency of morph $i$ with trait $T_{i}$ at temperature $T$ \\
\hline$n_{i}(x)^{\mathrm{a}}$ & Not applicable & Capture success of morph $i$ at depth $x$ \\
\hline$m(T)^{\mathrm{a}}$ & $\mathrm{g} \mathrm{s}^{-1} \mathrm{~m}^{-1}$ & Maintenance-cost density at temperature $T$ \\
\hline$B_{i}(x)$ & $\mathrm{g} \mathrm{m}^{-1}$ & Biomass of morph $i$ at depth $x$ \\
\hline$B_{i}$ & g & Total biomass of morph $i$ \\
\hline$b_{i}$ & g & Average biomass of an individual of morph $i$ \\
\hline$f_{i}(x)$ & $\mathrm{g} \mathrm{s}^{-1} \mathrm{~m}^{-1}$ & Growth-rate density of morph $i$ at depth $x$ \\
\hline$f_{i}$ & $\mathrm{~g} \mathrm{~s}^{-1}$ & Invasion fitness of morph $i$ \\
\hline$g_{i}$ & $\mathrm{~g} \mathrm{~s}^{-1}{ }^{\circ} \mathrm{C}^{-1}$ & First derivative of invasion fitness at trait value of morph $i$ \\
\hline$h_{i}$ & $\mathrm{~g} \mathrm{~s}^{-1}{ }^{\circ} \mathrm{C}^{-2}$ & Second derivative of invasion fitness at trait value of morph $i$ \\
\hline
\end{tabular}

${ }^{a}$ Model input functions were calibrated from empirical data as specified in appendix B, available online.

Table A2: Model parameters

\begin{tabular}{|c|c|c|c|c|}
\hline Notation & Unit & Value $( \pm S E)^{\mathrm{a}}$ & Description & Sources \\
\hline$\alpha$ & Not applicable & 6.0 & Degree of foraging optimality & 1 \\
\hline$x_{\max }$ & $\mathrm{m}$ & 68 & Depth of lake bottom & 2 \\
\hline$T_{\min }$ & ${ }^{\circ} \mathrm{C}$ & $4.42( \pm .14)$ & Minimum temperature at lake bottom & 3 \\
\hline$T_{\text {diff }}$ & ${ }^{\circ} \mathrm{C}$ & $7.60( \pm .26)$ & Temperature difference between lake surface and lake bottom & 3 \\
\hline$T_{\max }$ & ${ }^{\circ} \mathrm{C}$ & 9.0 & Temperature below which the fish forage & 3 \\
\hline$\phi$ & $\mathrm{m}^{-2}$ & $.0073( \pm .0006)$ & Measure of how quickly temperature decreases with depth & 3 \\
\hline$Z_{\min }$ & $\mathrm{g} \mathrm{m}^{-1}$ & .057 & Minimum zooplankton density at & 4 \\
\hline$Z_{\text {diff }}$ & $\mathrm{g} \mathrm{m}^{-1}$ & .78 & Difference in zooplankton density between lake surface and lake bottom & 4 \\
\hline$\varphi$ & $\mathrm{m}^{-1}$ & .047 & Measure of how quickly zooplankton density decreases with depth & 4 \\
\hline$r_{\mathrm{P}}$ & day $^{-1}$ & .20 & Renewal rate of zooplankton & 5 \\
\hline$r_{\min }$ & Not applicable & .20 & Minimum foraging efficiency & 7 \\
\hline$v$ & ${ }^{\circ} \mathrm{C}^{-2}$ & .030 & Measure of how quickly foraging efficiency decreases around $T_{i}$ & 7 \\
\hline$C_{\max }$ & day $^{-1}$ & .040 & Maximum consumption rate & 8 \\
\hline$\psi$ & $\mathrm{m}^{-1}$ & .050 & Measure of how quickly capture success decreases with depth & 9 \\
\hline$\lambda$ & Not applicable & .6 & Conversion factor from zooplankton mass to fish mass & 10 \\
\hline$\gamma$ & $\mathrm{g} \mathrm{J}^{-1}$ & .00018 & Conversion factor from energy to mass & 6 \\
\hline$m_{0}$ & $\mathrm{~g}^{-\beta} \mathrm{day}^{-1} \mathrm{~m}^{-1} \mathrm{~J}$ & $.82( \pm .13)$ & Metabolic scaling factor & 7 \\
\hline$B_{\text {mean }}$ & $\mathrm{g}$ & 10 & Mean biomass of fish & 4,7 \\
\hline$\beta$ & Not applicable & $.93( \pm .03)$ & Metabolic scaling exponent & 7 \\
\hline$\omega$ & ${ }^{\circ} \mathrm{C}^{-1}$ & $.070( \pm .006)$ & Temperature coefficient of metabolism & 7 \\
\hline
\end{tabular}

Sources: 1. Figure B1, available online; 2. Mehner et al. 2005b; 3. Mehner et al. 2010a; 4. Helland et al. 2007; I. P. Helland, unpublished data; 5. Schulz et al. 2004; 6. Gjelland 2008; 7. Ohlberger et al. 2007; 8. Binkowski and Rudstam 1994; 9. Ohlberger et al. $2008 b$; 10. Ohlberger et al. 2011.

a Standard errors could be provided for only some of the empirically estimated parameters, either because of low numbers of data points or because estimates were taken from the literature. 


\section{Literature Cited}

Binkowski, F. P., and L. G. Rudstam. 1994. Maximum daily ration of Great Lakes bloater. Transactions of the American Fisheries Society 123:335-343.

Bolnick, D. I. 2004. Can intraspecific competition drive disruptive selection? an experimental test in natural populations of sticklebacks. Evolution 58:608-618.

Bolnick, D. I., and B. M. Fitzpatrick. 2007. Sympatric speciation: models and empirical evidence. Annual Review of Ecologv, Evolution, and Systematics 38:459-487.

Bolnick, D. I., and O. L. Lau. 2008. Predictable patterns of disruptive selection in stickleback in postglacial lakes. American Naturalist 172:1-11.

Bulmer, M. G. 1980. The mathematical theory of quantitative genetics. Clarendon, Oxford.

Coyne, J. A., and H. A. Orr. 2004. Speciation. Sinauer, Sunderland, MA.

Dieckmann, U., and M. Doebeli. 1999. On the origin of species by sympatric speciation. Nature 400:354-357.

Dieckmann, U., and R. Law. 1996. The dynamical theory of coevolution: a derivation from stochastic ecological processes. of Mathematical Biology 34:579-612.

Dieckmann U., J. A. J. Metz, M. Doebeli, and D. Tautz. 2004. Adaptive speciation. Cambridge University Press, Cambridge.

Diekmann, O. 2004. A beginner's guide to adaptive dynamics. Pages 47-86 in R. Rudnicki, editor. Mathematical modelling of population dynamics. Banach Center Publications, no. 63. Polish Academy of Sciences, Warsaw.

Doebeli, M., and U. Dieckmann. 2003. Speciation along environmental gradients. Nature 421:259-264.

- 2005. Adaptive dynamics as a mathematical tool for studying the ecology of speciation processes. Journal of Evolutionary Biology 18:1194-1200.

Enberg, K., C. Jørgensen, E. S. Dunlop, M. Heino, and U. Dieckmann. 2009. Implications of fisheries-induced evolution for stock rebuilding and recovery. Evolutionary Applications 2:394-414.

Gavrilets, S. 2005. "Adaptive speciation"-it is not that simple: a reply to Doebeli et al. Evolution 59:696-699.

Gavrilets, S., and A. Vose. 2007. Case studies and mathematical models of ecological speciation. 2. Palms on an oceanic island. Molecular Ecology 16:2910-2921.

Gavrilets, S., A. Vose, M. Barluenga, W. Salzburger, and A. Meyer. 2007. Case studies and mathematical models of ecological speciation. 1. Cichlids in a crater lake. Molecular Ecology 16:28932909.

Geritz, S. A. H., M. Gyllenberg, F. J. A. Jacobs, and K. Parvinen. 2002. Invasion dynamics and attractor inheritance. Journal of Mathematical Biology 44:548-560.

Geritz, S. A. H., E. Kisdi, G. Meszéna, and J. A. J. Metz. 1998. Evolutionarily singular strategies and the adaptive growth and branching of the evolutionary tree. Evolutionary Ecology 12:3557.

Gíslason, D., M. Ferguson, S. Skúlason, and S. S. Snorrason. 1999. Rapid and coupled phenotypic and genetic divergence in Icelandic Arctic char (Salvelinus alpinus). Canadian Journal of Fisheries and Aquatic Sciences 56:2229-2234.

Gjelland, K. Ø. 2008. Ecological interactions, light responses and vertical habitat use in a subarctic pelagic freshwater community. $\mathrm{PhD}$ thesis. University of Tromsø, Norway.
Heinz, S. K., R. Mazzucco, and U. Dieckmann. 2009. Speciation and the evolution of dispersal along environmental gradients. Evolutionary Ecology 23:53-70.

Helland, I. P., J. Freyhof, P. Kasprzak, and T. Mehner. 2007. Temperature sensitivity of vertical distributions of zooplankton and planktivorous fish in a stratified lake. Oecologia (Berlin) 151:322330.

Helland, I. P., C. Harrod, J. Freyhof, and T. Mehner. 2008. Coexistence of a pair of pelagic planktivorous coregonid fish. Evolutionary Ecology Research 10:373-390.

Helland, I. P., L. A. Vøllestad, J. Freyhof, and T. Mehner. 2009. Morphological differences between two ecologically similar sympatric fishes. Journal of Fish Biology 75:2756-2767.

Hilton, Z., Wellenreuther, M., and K. D. Clements. 2008. Physiology underpins habitat partitioning in a sympatric sister-species pair of intertidal fishes. Functional Ecology 22:1108-1117.

Houle, D. 1992. Comparing evolvability and variability of quantitative traits. Genetics 130:195-204.

Hudson, A. G., P. Vonlanthen, R. Müller, and O. Seehausen. 2007. The geography of speciation and adaptive radiation in coregonines. Advances in Limnology 60:111-146.

Ingram, T. 2011. Speciation along a depth gradient in a marine adaptive radiation. Proceedings of the Royal Society B: Biological Sciences 278:613-618.

Ito, H. C., and U. Dieckmann. 2007. A new mechanism for recurrent adaptive radiations. American Naturalist 170:E96-E111.

Jonsson, B., and N. Jonsson. 2001. Polymorphism and speciation in Arctic charr. Journal of Fish Biology 58:605-638.

Keller, I., and O. Seehausen. 2012. Thermal adaptation and ecological speciation. Molecular Ecology 21:782-799.

Kirkpatrick, M., and N. H. Barton. 1997. Evolution of a species' range. American Naturalist 150:1-23.

Knudsen, R., A. Klemetsen, P.-A. Amundsen, and B. Hermansen. 2006. Incipient speciation through niche expansion: an example from the Arctic charr in a subarctic lake. Proceedings of the Roval Society B: Biological Sciences 273:2291-2298.

Kottelat, M., and J. Freyhof. 2007. Handbook of European freshwater fishes. Kottelat, Berlin.

Larsson, S. 2005. Thermal preference of Arctic charr, Salvelinus alpinus, and brown trout, Salmo trutta: implications for their niche segregation. Environmental Biology of Fishes 73:89-96.

Leimar, O., M. Doebeli, and U. Dieckmann. 2008. Evolution of phenotypic clusters through competition and local adaptation along an environmental gradient. Evolution 62:807-822.

Lu, G., and L. Bernatchez. 1999. Correlated trophic specialization and genetic divergence in sympatric lake whitefish ecotypes (Coregonus clupeaformis): support for the ecological speciation hypothesis. Evolution 53:1491-1505.

Mayr, E. 1963. Animal species and evolution. Belknap, Cambridge, MA.

Mehner, T., S. Busch, I. P. Helland, M. Emmrich and J. Freyhof. 2010a. Temperature-related nocturnal vertical segregation of coexisting coregonids. Ecology of Freshwater Fish 19:408-419.

Mehner, T., M. Diekmann, U. Brämick, and R. Lemcke. 2005a. Composition of fish communities in German lakes as related to lake morphology, trophic state, shore structure and human use intensity. Freshwater Biology 50:70-85.

Mehner, T., F. Hölker, and P. Kasprzak. 2005b. Spatial and temporal heterogeneity of trophic variables in a deep lake as reflected by repeated singular samplings. Oikos 108:401-409. 
Mehner, T., K. Pohlmann, C. Elkin, M. T. Monaghan, B. Nitz, and J. Freyhof. 2010b. Genetic population structure of sympatric and allopatric populations of Baltic ciscoes (Coregonus albula complex, Teleostei, Coregonidae). BMC Evolutionary Biology 10:85.

Mehner, T., S. Schiller, G. Staaks, and J. Ohlberger. 2011. Cyclic temperatures influence growth efficiency and biochemical body composition of vertically migrating fish. Freshwater Biology 56: $1554-1566$.

Metz, J. A. J., S. A. H. Geritz, G. Meszéna, F. J. A. Jacobs, and J. S. Van Heerwaarden. 1996. Adaptive dynamics: a geometrical study of the consequences of nearly faithful reproduction. Pages 183231 in S. J. Van Strien and S. M. V. Lunel, eds. Stochastic and spatial structures of dynamical systems. North Holland, Amsterdam.

Metz, J. A. J., R. M. Nisbet, and S. A. H. Geritz. 1992. How should we define fitness for general ecological scenarios? Trends in Ecology and Evolution 7:198-202.

Mizera, F., and G. Meszéna. 2003. Spatial niche packing, character displacement and adaptive speciation along an environmental gradient. Evolutionary Ecology Research 5:363-382.

Nosil, P. 2012. Ecological speciation. Oxford University Press, Oxford.

Ohlberger, J., E. Edeline, L. A. Vøllestad, N. C. Stenseth, and D. Claessen. 2011. Temperature-driven regime shifts in the dynamics of size-structured populations. American Naturalist 177:211-223.

Ohlberger, J., T. Mehner, G. Staaks, and F. Hölker. 2008 $a$. Is ecological segregation in a pair of sympatric coregonines supported by divergent feeding efficiencies? Canadian Journal of Fisheries and Aquatic Sciences 65:2105-2113.

. 2008b. Temperature-related physiological adaptations promote ecological divergence in a sympatric species pair of temperate freshwater fish, Coregonus spp. Functional Ecology 22:501-508.

Ohlberger, J., G. Staaks, and F. Hölker. 2007. Effects of temperature, swimming speed and body mass on standard and active metabolic rate in vendace (Coregonus albula). Journal of Comparative Physiology B 177:905-916.

Ohlberger, J., G. Staaks, T. Petzoldt, T. Mehner, and F. Hölker. 2008c. Physiological specialization by thermal adaptation drives ecological divergence in a sympatric fish species pair. Evolutionary Ecology Research 10:1173-1185.

Orr, M. R., and T. B. Smith. 1998. Ecology and speciation. Trends in Ecology and Evolution 13:502-506.

Østbye, K., T. F. Næsje, L. Bernatchez, O. T. Sandlund, and K. Hindar. 2004. Morphological divergence and origin of sympatric populations of European whitefish (Coregonus lavaretus L.) in Lake Femund, Norway. Iournal of Evolutionary Biology 18:683-702.

Pörtner, H. O., L. Peck, and G. Somero. 2007. Thermal limits and adaptation in marine Antarctic ectotherms: an integrative view. Philosophical Transactions of the Royal Society B: Biological Sciences 362:2233-2258.

Pörtner, H. O., P. M. Schulte, C. M. Wood, and F. Schiemer. 2010. Niche dimensions in fishes: an integrative view. Physiological and Biochemical Zoology 83:808-826.

Räsänen, K., and A. P. Hendry. 2008. Disentangling interactions between adaptive divergence and gene flow when ecology drives diversification. Ecology Letters 11:624-636.

Roughgarden, J. 1979. Theory of population genetics and evolutionary ecology. Macmillan, New York.
Schluter, D. 2000. The ecology of adaptive radiation. Oxford University Press, Oxford.

- 2003. Frequency dependent natural selection during character displacement in sticklebacks. Evolution 57:1142-1150.

- 2009. Evidence for ecological speciation and its alternative. Science 323:737-741.

Schluter, D., and J. D. McPhail. 1993. Character displacement and replicate adaptive radiation. Trends in Ecology and Evolution 8: 197-200.

Schulz, M., and J. Freyhof. 2003. Coregonus fontanae, a new springspawning cisco from Lake Stechlin, northern Germany (Salmoniformes: Coregonidae). Ichthyological Exploration of Freshwaters 14:209-216.

Schulz, M., J. Freyhof, R. Saint-Laurent, K. Østbye, T. Mehner, and L. Bernatchez. 2006. Evidence for independent origin of two spring-spawning ciscoes (Salmoniformes: Coregonidae) in Germany. Journal of Fish Biology 68(suppl. A):119-135.

Schulz, M., R. Koschel, C. Reese, and T. Mehner. 2004. Pelagic trophic transfer efficiency in an oligotrophic, dimictic deep lake (Lake Stechlin, Germany) and its relation to fisheries yield. Limnologica 34:264-273.

Snowberg, L. K., and D. I. Bolnick. 2008. Assortative mating by diet in a phenotypically unimodal but ecologically variable population of stickleback. American Naturalist 172:733-739.

Somero, G. N. 2002. Thermal physiology and vertical zonation of intertidal animals: optima, limits, and costs of living. Integrative and Comparative Biology 42:780-789.

. 2005. Linking biogeography to physiology: evolutionary and acclimatory adjustments of thermal limits. Frontiers in Zoology 2:1.

Svanbäck, R., and D. I. Bolnick. 2007. Intraspecific competition drives increased resource use diversity within a natural population. Proceedings of the Roval Society B: Biological Sciences 274:839-844.

Svensson, E. I. 2012. Non-ecological speciation, niche conservatism and thermal adaptation: how are they connected? Organisms, Diversity and Evolution 12:229-240.

Swanson, B. O., A. C. Gibb, J. C. Marks, and D. A. Hendrickson. 2003. Trophic polymorphism and behavioral differences decrease intraspecific competition in a cichlid, Herichthys minckleyi. Ecology 84:1441-1446.

Vamosi, S. M. 2003. The presence of other fish species affects speciation in threespine sticklebacks. Evolutionary Ecology Research 5:717-730.

van Tienderen, P. H., and G. de Jong. 1986. Sex ratio under the haystack model: polymorphism may occur. Journal of Theoretical Biology 122:69-81.

Via, S. 2001. Sympatric speciation in animals: the ugly duckling grows up. Trends in Ecology and Evolution 16:381-390.

Vonlanthen, P., D. Roy, A. G. Hudson, R. Largiadèr, D. Bittner, and O. Seehausen. 2009. Divergence along a steep ecological gradient in lake whitefish (Coregonus sp.). Journal of Evolutionary Biology 22:498-514.

Waxman, D., and S. Gavrilets. 2005. 20 questions on adaptive dynamics. Journal of Evolutionary Biology 18:1139-1154.

Associate Editor: Daniel I. Bolnick Editor: Mark A. McPeek 